\title{
Le paysage thanatique et féminin chez Gustave Moreau. Étude mythocritique de Orphée sur la tombe d'Eurydice
}

\author{
María FLORES-FERNÁNDEZ \\ Universidad de Granada \\ mflores@ugr.es \\ https://orcid.org/oooo-0003-0100-8146
}

\section{Resumen}

Este artículo propone definir el paisaje «tanático» como manifestación del arquetipo Hermes-Mercurio en el universo simbólico de Gustave Moreau, concretamente en Orphée sur la tombe d'Eurydice (1891) y sus escritos conservados. Este se define de acuerdo con la dualidad que tan presente está en la obra pictórica y literaria de Moreau; entre el texto y la imagen, lo tangible y lo espiritual, lo sagrado y lo profano, lo masculino y lo femenino. ¿De qué forma los elementos paisajísticos, humanos y sepulcrales, de origen religioso y mitológico, se fusionan y se confunden en el imaginario del pintor literario decadentista? En respuesta, este estudio adopta un enfoque mitocrítico y pretende aplicar la teoría arquetípica a la hermenéutica simbólica del paisaje.

Palabras clave: literatura francesa, antropología simbólica, arquetipo, mitocrítica

\section{Résumé}

Cet article propose de définir le paysage thanatique comme la manifestation de l'archétype Hermès-Mercure dans l'univers symbolique de Gustave Moreau, concrètement dans Orphée sur la tombe d'Eurydice (1891) et ses écrits conservés. Celle-ci est définie en accord avec la dualité si présente dans l'œuvre picturale et littéraire de Moreau ; entre le texte et l'image, le tangible et le spirituel, le sacré et le profane, le masculin et le féminin. Comment les éléments paysagers, humains et sépulcraux, d'origine religieuse et mythologique, s'unifient dans l'imaginaire du peintre littéraire décadent ? En guise de réponse, cette étude comporte une approche mythocritique et vise à appliquer l'archétypologie à l'herméneutique symbolique du paysage.

Mots-clés : littérature française, anthropologie symbolique, archétype, mythocritique

\section{Abstract}

\footnotetext{
${ }^{*}$ Artículo recibido el 7/06/2021, aceptado el 31/07/2021.
} 
This article proposes to define the thanatic landscape as the manifestation of Hermes-Mercurius archetype within the symbolic universe of Gustave Moreau, specifically on Orphée sur la tombe d'Eurydice (1891) and his preserved writings. This is defined according to the duality that is so present in Moreau's pictorial and literary work; between the text and the image, the tangible and the spiritual, the sacred and the profane, the masculine and the feminine. How do the human and sepulchral landscape elements, of religious and mythological origin, merge in the imaginary of the decadent literary painter? In response, this study includes a myth criticism approach and aims to apply the archetypal theory to the symbolic hermeneutics of landscape.

Keywords: french literature, symbolic anthropology, archetype, myth criticism

\section{Introduction}

Le paysage funéraire témoigne la confluence des cultures et des traditions liées à la mort qui survivent dans l'imaginaire collectif, à tel point que ces espaces évocateurs sont récurrents dans des œuvres francophones appartenant au domaine de la littérature, de la peinture ou de la sculpture ${ }^{1}$. Tout en considérant le cimetière comme un lieu syncrétique où s'encadre le paysage «thanatique » (Delcroix ; Hallyn, 1982: 9), il est possible de découvrir, sous sa structure polymorphe, une source de symboles autour de l'imagerie de la mort. En ce qui concerne son caractère non seulement féminin, mais hermétique, nous entendons ici le paysage en tant qu'espace relayant les différents aspects de Thanatos, du mythe et de sa symbolique. Le but de cette étude est d'élucider la féminisation du paysage mystique en tant qu'élément exponentiel de la dimension mythico-religieuse chez Gustave Moreau, né à Paris en 1826 et considéré comme un peintre romantique tardif. De fait, dans la préface du catalogue de Ragnar von Holten à propos de Gustave Moreau, Jean Cassou affirme que « cet artiste est né trop tôt ou trop tard » (1960 : 30) pour le définir en concordance avec les mouvements littéraires et artistiques de la fin de siècle. Contemporain de Pierre Puvis de Chavannes et influencé par les couleurs lumineuses de Théodore Chassériau, ainsi que par l'orientalisme et la mort ${ }^{2}$ chez Eugène Delacroix, le système d'opposition et les images par inversion dans l'œuvre de ce peintre passionné de mythologie, ne sont pas seulement représentés sous une intention esthétique singulière, mais ils témoignent une dimension mythique et psychique du paysage littéraire et pictural. Ainsi, la couleur qui a communément dominé la représentation du paysage de Moreau est le vert foncé, parsemé de clair-obscur, de violet et de bleu - « terre d'ombre et terre verte,

${ }^{1}$ Il est à remarquer Le Cimetière marin (1920) de Paul Valéry, Jeune orpheline au cimetière (1824) de Eugène Delacroix et la sculpture Le Baiser (1909) de Constantin Brâncusi, sur la tombe de Tatiana Rachewskaïa, au cimetière de Montparnasse.

${ }^{2}$ La mort de Sardanapale (1827). Huile sur toile, $392 \times 496 \mathrm{~cm}$. Musée du Louvre, Paris. 
ocre derrière. Montagnes violettes ou bleu pervenche. [...] Montagnes bleues, dorées avec poussière d'or les réunissant au ciel » (Cooke, 2002 : 272) ; des couleurs de deuil, des couleurs de mort dont l'artiste a imprégné ses huiles et ses écrits personnels lors d'une période turbulente, marquée par la perte de ses proches et par une quête identitaire.

À partir des oppositions propres à la structure synthétique de l'image, selon la théorie de Gilbert Durand, herméneute et anthropologue français visant à définir le sémantisme de l'imaginaire, des images d'inversion par conversion et réduplication sont présentes dans l'œuvre de Moreau. Pour Durand, l'imaginaire est conçu en fonction de deux régimes de l'image qui se situent sous le signe d'une dualité complémentaire : d'un côté, un régime diurne en lien avec les images lumineuses, l'antithèse de la lumière et les ténèbres, la lutte contre le temps et la mort ; d'un autre côté, un régime nocturne axé sur l'euphémisme, qui se concentre sur la volonté d'union des contraires - structure synthétique - et le goût de l'intime - structure mystique. Cette deuxième structure se définit comme une dimension dans le domaine de l'imaginaire où l'on assiste à une conjugaison de Kronos, d'Éros et de Thanatos. Sur la base de l'affirmation précédente, le régime nocturne génère des images symboliques qui coexistent, même si elles sont dissonantes et contraires. Dans ce sens, le mélange d'éléments entraine des processus d'inversion, de réinvestissement et de conjonction des contraires qui sont très présents dans la sphère picturale et littéraire. Plus précisément, ce carrefour dialectique est défini dans Orphée sur la tombe d'Eurydice (1891), ainsi que dans les annotations et pensées de Moreau, pieusement conservées et ressemblées par Peter Cooke dans l'ouvrage Écrits sur l'art par Gustave Moreau (2002).

La présente étude s'appuie sur ce corpus pour démontrer, comme souligne Annick Béague (1998 : 34), que «l'histoire des interprétations du mythe d'Orphée donne à lire une partie de l'histoire de la culture européenne, comme on le constate pour tous les grands mythes classiques ». C'est ainsi que la rupture des tabous esthétiques et moraux à travers la relation d'éléments opposés et liés à la mythologie et à la religion reflète la nature cyclique des mythes et des civilisations, concrètement dans un paysage funéraire et apparemment féminin. Dans cet œuvre, où la nature semble être à l'état latent, l'hermétisme est néanmoins dessiné en accord avec l'ambivalence omniprésente dans l'œuvre de Moreau : le tombeau d'Eurydice en tant que porte vers l'enfer, face à un Orphée asexué et analogue à la figure du Christ (voir Charbonneau-Lassay, 1925). En illustrant l'interaction d'univers contraires, Gustave Moreau crée deux dimensions antithétiques dans la structure de nos sociétés occidentales. Comment les éléments paysagers, humains et sépulcraux, d'origine religieuse et mythologique, s'unifient dans l'imaginaire de ce peintre littéraire ? En matière des sciences de l'imaginaire, cet espace est fortement marqué par la symbolique de l'inconscient, 
du féminin et du maternel. Pour développer cette perspective, il est nécessaire de s'appuyer non seulement sur les notes du peintre, mais aussi de recourir à la psychanalyse et aux régimes de l'image durandiens, notamment le régime nocturne comportant des « syntaxes d'inversion et de répétition » (Durand, 2016 [1960] : 293). Ces outils permettront de définir le paysage de mort en tant que symbole hermétique chez Gustave Moreau, tout en donnant une réponse à la pérennité de la figure d'Orphée et d'Eurydice, le pôle masculin et le pôle féminin, à travers leur jonction dans l'archétype de Hermès-Mercure. Celui-ci commence à se réactiver dans les grands thèmes de la scène littéraire et picturale à la fin du XIX ${ }^{\mathrm{e}}$ siècle en France : l'alchimies, l'androgynie et la mort (voir Praz, 1977).

\section{Du récit mythique à la représentation plastique}

\footnotetext{
3 Les ouvrages des alchimistes en Occident deviennent de plus en plus nombreux à partir du XIII siècle, mais après un déclin important au XVII e siècle, une résurgence de l'alchimie a lieu au $\mathrm{XIX}^{\mathrm{e}}$ et $\mathrm{XX}^{\mathrm{e}}$ siècle par le biais de la réédition des traités et manuscrits anciens et de nombreuses parutions inspirées de la vision symbolique de l'univers au sein de la tradition occultiste, ravivée sous le prestige de la logique empirique. Les auteurs français de la seconde moitié du XIX ${ }^{\mathrm{e}}$ siècle sont attirés par les idées, les mythologies et les pratiques occultes qu'Éliphas Lévi, Papus et Stanislas de Guaita ont popularisées. De Baudelaire et Verlaine, Lautréamont et Rimbaud, à André Breton, ces artistes ont intégré l'alchimie dans leurs discours artistiques et littéraires, avides de nouveaux idéaux esthétiques.
} 
Le paysage funéraire comporte tout un aspect mythique et psychique donnant lieu à une constellation de symboles qui traverse différentes époques, notamment les imaginaires décadents. Étant donné que «les cimetières sont devenus des lieux de promenade publique au XIX ${ }^{\mathrm{e}}$ siècle » (Maingon, $2015:$ 179), Gustave Moreau décide de recréer cet espace dans Orphée sur la tombe d'Eurydice, l'une de ses peintures mythologiques. Il

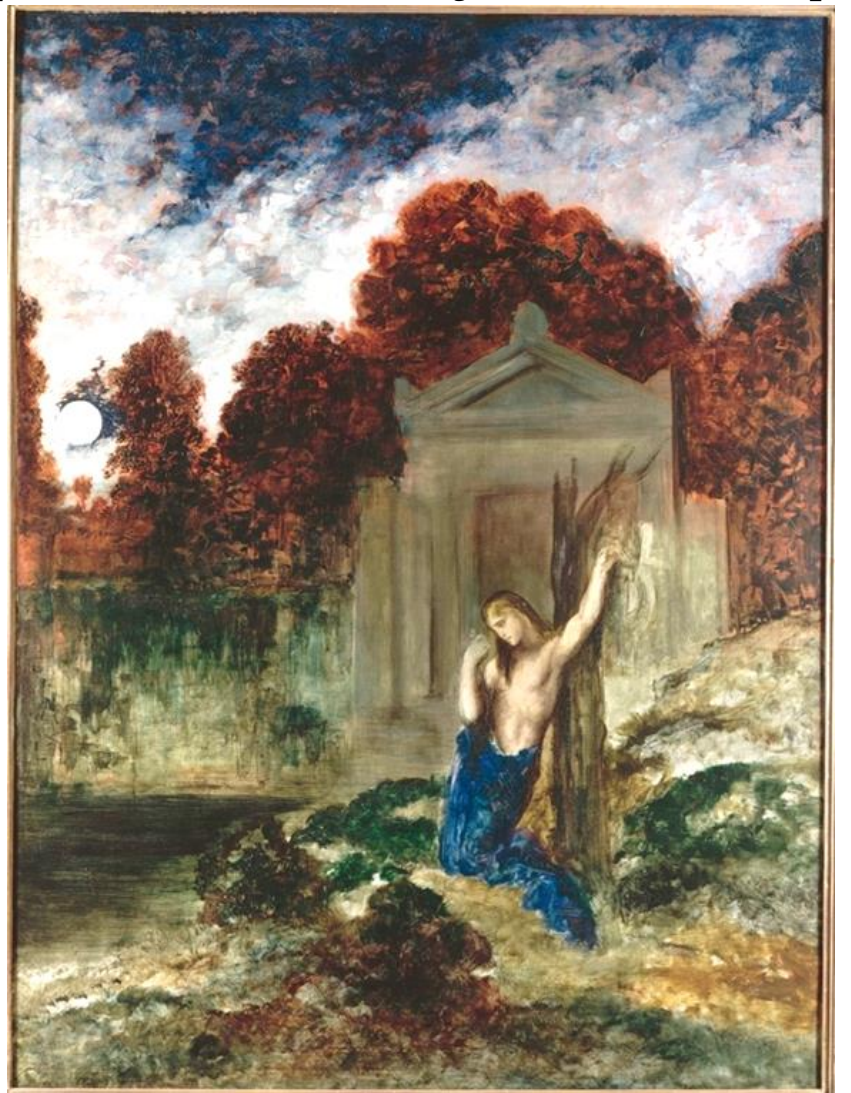

Gustave Moreau, Orphée sur la tombe d'Eurydice, 1891. est à cheval entre la tradition romantique, pour qui le paysage est extérieur et exprime un lieu d'expansion de l'être, et symboliste, pour qui le paysage n'existe que sous une forme intérieure, comme un miroir de l'âme. En ce sens, le peintre associe le paysage de mort à un trajet à travers les évènements et les pertes qu'il a subies au cours des dernières années de sa vie - plus précisément, le décès de ses parents en 1862 et 1884, et de Alexandrine Dureux, sa «meilleure et unique Huile sur toile, 173 x $128 \mathrm{~cm}$. Musée Gustave Moreau, Paris. amie ». C'est ainsi qu'un paroxysme important commence à être présent dans ses œuvres.

L'œuvre qui reprend le mythe d'Orphée et Eurydice ne répond qu'au thanatisme - néologisme emprunté à Jonathan Littel4 - qui détermine la production littéraire et artistique de Moreau, mais il révèle aussi son univers symbolique dans son approche la plus intime d'un paysage funéraire féminisé. Comme s'il était un décor d'une identité à reconstituer et d'une résonance autobiographique, l'interprétation du cimetière dans la toile répond à l'expression des mythes personnels de l'auteur dans un paysage dont l'atmosphère et les personnages sont marqués par le deuil. Moreau rend ainsi hommage à Adelaïde-Alexandrine Dureux, décédée le 28 mars 1890, pour

4 Éros et Thanatos se rejoignent chez Littell en tant que deux « imaginaires » et deux « jeux de hantises ressassés » (Littell, $2006:$ 810). 
qui il partageait un lien intime et platonique. Contrairement à Eugène Delacroix (1986 [1829]), il a toujours refusé de publier ses écrits et ses réflexions personnelles, car « il ne reconnaissait pas aux écrivains le droit de juger la peinture » (Cooke, 2002 : 280). Les notes qui accompagnent ses toiles et ses dessins, et qui ont été occultés pendant trente ans, constituent aujourd'hui un corpus littéraire qui lui donne, après son existence, le statut d'un écrivain capable de traduire l'histoire mythique en représentation plastique, de transposer le mythe ancien à l'expression romantique et décadente du mundus imaginalis (Corbin 1983 [1964]), malgré l'essor des sciences positivistes :

Distinction entre les mythes traduits par les anciens ou par les modernes, les mystères antiques et les mystères modernes : les premiers relèvent des grands phénomènes généraux de la nature tandis que les nôtres relèvent de l'intimité et de la profondeur du sentiment, la pensée chrétienne dominant tout chez nous, malgré toutes les théories positivistes (Cooke, $2002: 231$ ).

Suite à la mort d'Alexandrine Dureux, il exécute à sa mémoire un dessin préparatoire faisant directement allusion à celle-ci dans le détail de l'œuvre : « Orphée, à la mémoire de la chère bien aimée A. D. » (Cooke, 2002 : 174). Cette composition est inspirée par le mythe d'Orphée et Eurydice dans les Métamorphoses d'Ovide (Livre X, 1-105), récit que l'auteur reprend à plusieurs reprises - en 1865, il conçoit Orphée, ouvrage conservé au Musée d'Orsay5 -. Ce mythe décrit comment la dryade, lors d'une promenade en compagnie d'une troupe de naïades est attaquée par un serpent le jour même de ses noces. Une morsure au talon provoque sa mort et en conséquence, son époux Orphée, entraîné par la douleur, descend aux Enfers et traverse la lagune Stygienne, qui divise le royaume de la lumière et le royaume des ténèbres, pour implorer d'Hadès, le souverain des ombres, et de Perséphone, fille de Zeus et de Déméter, la grâce de ramener Eurydice dans le monde des vivants. Ce vœu est accepté sous la seule condition de ne pas regarder en arrière avant d'avoir quitté les vallées de l'Averne. Mais, victime du désir insurmontable de revoir sa bien aimée, il enfreint l'interdit divin et la perd donc à jamais. C'est ce moment tragique que Moreau décide de réinterpréter en mettant le fils d'Eagre, roi de Thrace, et de la muse de

\footnotetext{
5 La toile Orphée ou Jeune fille thrace portant la tête d'Orphée (1865) montre une lecture différente du mythe, tout en donnant plus d'importance à la figure féminine et à l'image immortelle du poète. Si la fille de Thrace représente la poésie dont elle est la muse, les instruments tels que la lyre, la flûte, ainsi que les deux tortues qui représentent le couple d'Orphée et d'Eurydice situées au premier plan de la composition, évoquent le caractère éternel de la poésie et de la musique, même après la mort de l'artiste. Rappelons que la lyre d'Orphée est un attribut associé à Hermès, dieu chargé de la fabriquer à partir d'une carapace de tortue. Le tableau de Moreau donne ainsi au mythe d'Orphée le caractère éternel d'un temps victorieux face à la mort.
} 
Calliope, au centre d'un paysage aux teintes automnales et crépusculaires, et faisant ainsi preuve du lien entre la nature et les émotions, entre la littérature mythique et les arts plastiques.

Le transcodage des éléments mythiques, de l'expression littéraire à la peinture, aboutit à un effet d'assemblage sur les éléments de l'art récepteur. "Rien n'est moins fixé que le mythe », dit Pierre Brunel (1992 : 73) et c'est lui qui introduit la notion de flexibilité du mythe - ainsi que celle d'émergence et irradiation - car « dès les premières versions connues, on est surpris par le nombre et l'importance des variantes : Orphée est fils d'Apollon ou fils d'Eagre, marié ou non marié, victorieux de la mort ou vaincu par elle ». Faisant écho aux paroles du mythologue, José Manuel Losada (2013 : 11) explique que le mythe s'adapte habilement à un nouveau média, « un médium, pourrait-on dire, imprévu, puisque sa naissance naturelle a été littéraire ». En outre, " la flexibilité du mythe est telle qu'il peut être adapté à un autre art de la manière qui lui semble la plus appropriée » (Losada Goya \& Lipscomb, $2013: 11$ ), un axe que la mythocritique vise à étudier à travers l'interdisciplinarité. Dans la présente analyse, cette discipline est appliquée en tenant compte du fait que " pour comprendre la vie (l'évolution) des archétypes littéraires il faut les insérer dans les paradigmes religieux et culturels qui les sécrètent » (Braga, 2019 : 26). Ce phénomène est défini par Gilbert Durand comme des « images naturelles, suggérées par la situation psycho-physiologique » (Durand, 1996a : 39), et qui, dans ce cas précis, se trouvent dans le vécu de Moreau en tant que symboles païens et chrétiens. Aux dires de Durand (1996a : 39), « c'est ce qui explique l'universalité du fonds mythologique - et sa traductibilité -, la société ne faisant la plupart du temps que 'dériver' (selon le mot de Pareto) les grands schèmes et archétypes naturels qui structurent en son fond le mythe ». La tradition mythique-littéraire a besoin d'une application historique, en comprenant le mythe comme un universel, " un langage muet, qui aura pu prouver, le sublime inventeur, le savant, le divin artiste, que tout l'homme peut s'exprimer dans cette langue du symbole » (Cooke, 2002 : 258). En outre, aux dires de Gustave Moreau, il y a « un moment fatal où un art se transforme pour prendre les qualités des arts voisins » (2002 : 258). C'est pourquoi, la transmutation du langage textuel en langage iconique révèle un espace de création régi par le mythe, qui apparaît comme « une structure symbolique d'images, particulièrement apte à susciter et à diriger la création » (Wunenburger, 2005 : 69). Dans la même veine que les auteurs précédents, Jean-Jacques Wunenburger, philosophe de l'imaginaire, considère que « le mythe constitue une matrice archétypale à partir de laquelle l'imagination recrée, reconstruit de nouvelles histoires » (2005: 79-80). À cet égard, la relecture du mythe d'Orphée, sous la plume et le pinceau de Gustave Moreau, met en lumière des éléments pérennes, et aussi variables, dans son approche de l'archétype Hermès-Mercure, une figure médiatrice, par le biais des para- 
mètres iconographiques. Toujours contemporain, il a emporté leur puissance symbolique dès la fin du XIX ${ }^{\mathrm{e}}$ siècle et reste fortement ancré dans l'inconscient de l'artiste. L'œuvre artistique et littéraire de Moreau met en évidence la richesse de l'intertextualité des images hermésiennes porteuses "des bornes, des limites qui définissent seules rencontres et carrefours » (Durand 1996c : 230). Hermès et Orphée sont les intermédiaires entre le ciel et la terre, entre le jour et la nuit, entre la vie et la mort, entre les deux sexes, mâle et femelle, et que Gustave Moreau unifie dans un paysage mythique.

\subsection{Résonances symboliques et iconographiques du mythe}

Le paysage est omniprésent dans les tableaux mythologiques de Gustave Moreau sous l'influence de ses prédécesseurs, de Léonard de Vinci à Claude Gellée, dit le Lorrain. Cependant, de nombreuses esquisses peuvent difficilement être reliées à des iconographies identifiables, à l'exception de quelques-unes d'entre elles : Gustave Moreau, tout en se libérant de sa douleur, réécrit les amours malheureux d'Orphée et d'Eurydice pour transposer son vécu à l'expression artistique et poser un nouveau regard sur un paysage hybride. Chez Moreau, la figure du poète tragique secouant les arbres et les montagnes avec ses chants est étroitement liée à l'intime et à la dualité. L'incorporation d'Orphée dans l'iconographie chrétienne répond à une représentation du Christ comme le nouvel Orphée, en raison de la puissance libératrice du chant ou des paroles. D'après Gilbert Durand, « quelquefois Orphée prend le nom de cet autre thrace Dionysos, d'autres fois encore c'est Jésus-Christ lui-même qui se coiffe du bonnet de Phrygie et empoigne la lyre » (1997 : 21). La mythanalyse se produit quand elle prolonge naturellement la mythocritique, c'est-à-dire, l'analyse des textes et des objets mythiques dans leur dimension diachronique, tout en abordant des "séquences et des mythèmes d'un mythe préétabli » (Durand, 1979 : 310). Donc, il est possible de déceler le mythe d'Orphée et Eurydice à travers l'étude des diverses reprises de son mythème central, unique et facilement repérable. Ce mythème est invariable dans toute version du mythe tandis que les mythèmes secondaires, " qualitatifs, mais quantitativement constants » (Durand 1996 : 104), ainsi que leur entité symbolique, pourraient changer d'une version à l'autre.

Durand montre que les groupes mythémiques d'Orphée, ses résonances et ses invariantes " gravitent autour de la nostalgie, du nostos (le retour) et algia, la douleur » (Durand, $1997: 21)$. Parallèlement, dans son identification intime, Moreau exprime à travers la figure d'Orphée l'exigence de son art et la conscience de son échec face à la société du XIX ${ }^{\mathrm{e}}$ siècle. C'est pourquoi Gustave Moreau a tendance à peindre les poètes androgynes, affligés ou morts, et non au temps de leur gloire. Le mythe d'Orphée, autant 
masculin que féminin, est récurrent dans différents domaines tout en suivant le cours du " bassin sémantique ${ }^{6}$ durandien, en musique - l'opéra Orphée et Eurydice (1774) de Gluck, un des compositeurs préférés de Moreau, et Orphée aux enfers d'Offenbach, en 1858 -, en littérature chez les auteurs romantiques - Orphée et Chiron (1847) de Leconte de Liste et dans le sonnet Orphée (1891) de Paul Valéry-. Certes, dans la plupart de ces manifestations culturelles, la figure d'Eurydice est souvent omise ou négligée7. Cependant, l'influence d'Orphée sur l'imaginaire collectif et principalement sur l'imaginaire individuel du peintre, invite celui-ci à transgresser le mythe tout en donnant à Eurydice plus de relevance dans son œuvre.

Par ailleurs, il convient de noter qu'étant la mort dans la poésie baroque possiblement perméable à la pensée de Gustave Moreau, Fernand Hallyn met en lumière les thèmes qu'à cette époque accompagnent la représentation de Thanatos, « aussi bien la thématique religieuse, depuis la stigmatisation de l'inconstance humaine jusqu'à la célébration de l'expérience mystique, que la thématique amoureuse » (Hallyn, 1982 : 10). Sous ce prisme, le paysage thanatique montre une nature dotée d'une identité féminine ainsi qu'un pouvoir de consolation, comme s'il s'agissait d'un "refuge » (Guhl, 1972 : 22), tout comme le ventre maternel que, dans la structure mystique, symbolise le lieu intime et féminin - la femme et la mère. La tombe euphémisée comme une grotte, une maison, un berceau ou le ventre maternel, représente le repos et la protection contre le temps implacable - Kronos - et la mort.

De même, Moreau intègre la figure de Christ dans l'iconographie mythologique, quand il l'assimile à Orphée et incorpore une Eurydice' au centre d'un paysage d'automne, réifiée dans une crypte funéraire, "symbole du souvenir et de la piété fidèle et invincible » (Cooke, $2002: 134$ ). La religion, spécifiquement chrétienne, est inscrite à la fois dans un paysage où le syncrétisme religieux et l'histoire mythique parviennent à coexister à travers la conciliation des contraires. Gustave Moreau, artiste non pratiquant, décide donc de représenter divers principes et personnages bibliques en relation directe avec les dieux de la mythologie gréco-romaine. Comme l'indique Mercedes Montoro Araque à propos de Théophile Gautier, dans l'œuvre de Gustave Moreau nous pouvons remarquer « un condensé de mythologie personnelle $[. .$.$] mais aussi l'illustration d'un désir syncrétique ». Parallèle-$

\footnotetext{
${ }^{6}$ Dans le champ des sciences de l'imaginaire, Gilbert Durand définit le bassin sémantique comme une « ère de quelque cent cinquante années environ où un air de famille, une isotopie, une homéologie commune, relie épistémologie, théories scientifiques, esthétique, genres littéraires, visions du monde » (Durand, 1996a : 81).

7 Orphée et Chiron, écrit par Leconte de Lisle en 1847, met en valeur un Orphée écoutant les enseignements du Centaure sur l'histoire de la Grèce, et sur une forme de sagesse méditative. Dans le sonnet de Paul Valéry paru en 1891, Eurydice est absente également, le texte étant centré sur la figure poétique d'Orphée.
} 
ment à cette toile, et en reprenant les mots de Grunewald sur les assimilations syncrétiques, "Orphée est en même temps Osiris, Psyché incarne aussi bien la Grecque Eurydice que la Vierge Marie » (Montoro Araque, 2019 : 323). Sous ce rapport, il est essentiel de souligner l'importance des associations chromatiques du bleu, la couleur de Marie dans le christianisme, et l'opposition entre mythologie et religion suivant la pensée de Leopold Stein, étant donné que la figure archétypale du messager est incarnée par Hermès ou Christ (voir Stein, 1967). Associer Orphée à Hermès, et corrélativement au Christ, renvoie à la façon dont Moreau commente l'une de ses œuvres, La Vie de l'Humanité (1886), en la divisant en trois phases correspondant aux grandes étapes de la vie de l'homme : "La pureté de l'enfance : Adam. Les aspirations poétiques et douloureuses de la jeunesse : Orphée. Les souffrances pénibles et la mort pour l'âge viril : Caïn, avec la rédemption par le Christ » (Cooke, 2002 : 127). D'après l'étude de Françoise Bonardel sur la figure d'Hermès, celui-ci est défini comme « l'esprit renaissant, lui-même épris de réconciliation, d'unification autant que d'éclectisme, de retour aux sources et de progrès » (Bonardel, 2011 : 82). De même que Gilbert Durand a établi quatre ensembles synchroniques du récit diachronique - la quête initiatique, le retour, les amours malheureux et la lyre - (Durand, 1997 : 22), Orphée sur la tombe d'Eurydice montre les trois phases définies par rapport à l'histoire mythique ainsi qu'à la vie de l'auteur, ou selon Moreau, à la vie humaine, au sens plus large. Ainsi, ces périodes sont associées aux trois âges de la mythologie sacrée et profane : l'enfance, la jeunesse et la maturité de l'homme. C'est à l'âge d'argent, "l'âge des poètes » (Cooke, $2002: 127)$ appartenant à la mythologie païenne, que le peintre situe Orphée comme la personnification de la nature : une nature sensorielle et créative, fondée sur le rêve ; une nature régénératrice et fascinée par le chant, et enfin, une nature qui aspire à l'éternité :

$1^{\circ}$ Le rêve : la nature se dévoile aux sens du poète ému, qui s'en inspire.

$2^{\mathrm{O}}$ Le chant : Orphée chante, la nature entière l'écoute et l'admire.

$3^{\mathrm{O}}$ Les pleurs : Orphée dans les grands bois, sa lyre brisée, aspire à des pays inconnus et à l'immortalité (Cooke, $2002:$ 126).

De plus, ce choix iconographique se justifie en accord avec la pensée de Bonardel qui, d'une perspective hermésienne, parallèlement aux « aspirations aussi contrastées, au point de paraître contradictoires, qui pouvait, sinon Hermès, apposer le sceau de son inépuisable créativité ? » (2011 : 89). Gustave Moreau, profondément marqué par une pensée hermétique, établit un jeu analogique des images contraires dans lequel il privilégie finalement un Orphée païen et médiateur, en laissant de côté la figure biblique :

Destouches a trouvé extrêmement ingénieux et intelligent d'avoir pris pour les cycles de la jeunesse et de la 
poésie une figure de l'antiquité païenne au lieu d'une figure biblique, parce que l'intelligence et la poésie sont bien mieux personnifiées dans ces époques tout entières d'art et d'imagination que dans la Bible, toute de sentiments et de religiosité (Écrits sur l'art, 2002 : 127).

Orphée sur la tombe d'Eurydice introduit une esthétique paradigmatique et le mélange du mythe païen et de l'histoire biblique. Ce syncrétisme se dévoile par le biais d'Orphée, une « figure de l'antiquité païenne » ayant une valeur inhérente à la fascination de l'artiste pour ce poète qui partage avec Hermès une passion pour la musique et la lyre. Cet instrument nous permet d'établir une passerelle vers la notion de paysage sonore (voir Murray Schaefer, 1979) - un environnement sonore, dans cas-là, pictural et littéraire -, car dans le tableau de Moreau l'espace orphique s'inscrit dans un paysage nocturne propre du règne des ombres qui devient chant. Il est présenté sous la forme d'une identité acoustique, des sons qui habitent à l'intérieur d'Orphée et chez Moreau. Ainsi, Renato Boccali soutient que ce héros " subit sa fascination et, séduit lui-même par son pouvoir enchanteur, il s'oublie à la recherche de la source inépuisable de ce chant, c'est-à-dire Eurydice » (2016 [1960] : 62). Du point de vue d'une lecture païenne du récit, ce pouvoir renvoie également à la « catastérisation » (Martin, 2002 : 17) de la constellation Lyra en lien avec le chant orphique. Après avoir manqué à sa promesse et avoir perdu Eurydice, Orphée se consacre au culte d'Apollon, dieu de l'Harmonie et des Arts, mais les prêtresses de Dionysos, envieuses de sa voix, lui donnent la mort. Néanmoins, son âme est recueillie dans l'île des Bienheureux et sa lyre est placée dans le cosmos. En effet, « l'âme d'Orphée pour l'éternité fut assignée pour charmer les Bienheureux de l'autre monde, tandis que la lyre à jamais est une constellation de notre ciel » (Durand, 1997 : 21). Il s'agit bien d'une « constellation d'images », au sens durandien, où plusieurs symboles convergent et des « déclinaisons » du mythe d'Orphée témoignent l'holisme cyclique des récits mythiques (Durand, 2016 [1960] : 22).

\subsection{Dimension mythique et psychique du paysage littéraire et pictural}

La construction du paysage thanatique dans l'œuvre de Moreau répond à des paramètres non seulement mythiques, mais psychiques. Parmi ses écrits personnels, il est à souligner des notes signées en 1897, dans lesquelles il décrit sur un ton tragique et romantique la toile qui concerne cette étude :

L'âme est maintenant seule, elle a perdu tout ce qui était sa splendeur, sa force et sa douceur. Elle pleure sur ellemême dans sa solitude inconsolée. Elle gémit et sa plainte sourde et sans éclat est le seul bruit d'humanité de cette solitude de nuit (Cooke, 2002 : 134). 
Cette âme silencieuse, en opposition au paysage sonore, et « sans éclat », renvoie à la notion donnée par Gaston Bachelard (1984:47) en lien avec l'image du paysage nocturne, à savoir, l'aspect diurne face à l'aspect nocturne de l'âme : " Trop tard, j'ai connu la bonne conscience dans le travail alterné des images et des concepts, deux bonnes consciences qui seraient celle du plein jour et celle qui accepte le côté nocturne de l'âme ». D'un côté, ces lignes introduisent la solitude du deuil, des sentiments transcodés à la peinture par le biais de la couleur bleuâtre des vêtements d'Orphée, symbole de tristesse présent dans Ovide chez les faux (1859) de Delacroix. Or, le bleu est également une couleur passive et féminine qui renvoie à la sensualité, à l'âme, au mystère et au secret. Le symbolisme traditionnel le lie à l'eau et à l'humidité, suivant la théorie des humeurs, comme attribut du corps féminin. En outre, dans la peinture chrétienne, elle est utilisée pour caractériser les vêtements de la Vierge Marie, étant communément appelée « la couleur de la Vierge ». Cette figure apparaît identifiée au lapis-lazuli le plus lumineux, l'un des pigments les plus précieux, et accompagnée de la lune, comme une déesse céleste. D'autre part, la position du corps d'Orphée constitue un triangle renversé, symbole passif et féminin. Ainsi, Gustave Moreau crée ses mythes personnels à travers une figure ambivalente faisant l'objet d'une dynamique d'inversion systématique qui fait écho également aux régimes de l'image de la théorie durandienne. Le paysage funéraire de cette image s'inscrit dans le régime nocturne que Gilbert Durand introduit dans Les structures anthropologiques de l'imaginaire (1960) et qui se fonde sur l'interaction d'éléments opposés et contextualisés dans la nuit. Ce régime se trouve donc « constamment sous le signe de la conversion et de l'euphémisme » (Durand, 2016 [1960] : 201), c'est-à-dire, il est déterminé par la métamorphose et l'existence d'oppositions dans la même unité. Il s'agit d'une dimension faisant appel au besoin de retrait et d'intimité, une pulsion de mort qui place le spectateur devant une image d'investissement par réduction $^{8}$ : Moreau incarné dans Orphée, ainsi qu'Alexandrine dans Eurydice ; deux images opposées qui se transforment en leur contraire sous un effet de mimétisme inversé. Ces personnages constituent la bi-unité d'un même sujet dans une seule image, où le pôle masculin et le pôle féminin s'entremêlent - compte tenu du fait que le visage et le torse d'Orphée ont une apparence androgyne - .

Revenant sur les mots d'Henri Rupp, très proche du peintre, il est possible de constater qu'au moment de la perte d'Alexandrine Dureux, ce dernier « a subi la disparition d'une âme, d'une créature exquise qu'il avait intégrée à son propre esprit au cours d'une relation indestructible de vingtcinq ans. Elle a emporté la moitié d'elle-même dans la tombe » (Brisson,

${ }^{8}$ En ce qui concerne le réductionnisme et le renversement des valeurs symboliques des images dans la réinterprétation du mythe par Moreau, Gilbert Durand (2016 [1960]) : 198souligne que « la duplicité permettant l'euphémisation de la mort elle-même ouvre à l'imagination et aux conduites qu'il motive une toute autre voie». 
1899 : 3). C'est pour cette raison que l'apparence physique d'Alexandrine est placée au deuxième plan. Cet effet est à mettre en rapport avec la « ligne de flottaison de l'imaginaire » de Jean Baudrillard (1987: 156) selon laquelle « certaines parties sont visibles, et d'autres non, les parties visibles rendent les autres visibles ; il s'installe une sorte de rythme de l'émergence et du secret ». De plus, dans le régime nocturne de l'image, Kronos n'est plus évident, comme il l'est dans le cimetière, lieu de mémoire. Ce propos est repris par Marcel Proust à propos des paysages de Moreau, "se trouvant généralement emprisonnés dans une grotte, entourés d'un lac », en l'occurrence, d'un étang sacré9, « là où le divin s'est parfois manifesté, à une heure incertaine que la toile éternise comme mémoire du héros » (Proust, 1971 : 668).

En outre, dans l'œuvre Orphée sur la tombe d'Eurydice, Moreau interprète l'élément eau dans le paysage mythique en relation avec le pouvoir cyclique et le caractère sacré, mais aussi comme la représentation du monde souterrain, de l'inconscient et d'une image intériorisée. Dans le cadre de la recherche interdisciplinaire, Florence Godeau soutient que « tout paysage, réel ou fictif, est la projection d'une image intériorisée » (Godeau, 2008 : 119). Ainsi, dans l'étude Notes sur le monde mystérieux de Gustave Moreau, Proust (1971 : 668) constate la conception des huiles en tant que fragments d'un monde intérieur propre à l'artiste :

Ces paysages de Moreau sont tellement le paysage où tel dieu passe, où telle vision apparaît, et la rougeur du ciel y paraît à côté bien vulgaire et comme désintellectualisé, comme si les montagnes, les ciels, les bêtes, les fleurs avaient été vidés en un instant de leur précieuse essence d'histoire, comme si le ciel, les fleurs, la montagne ne portaient plus le sceau d'une heure tragique, comme si la lumière n'était plus celle où passe le dieu.

La lumière introduit un moyen d'accéder à des symboles cachés - au régime diurne - contre « le mystère d'une crypte, la peur des ténèbres, de la nuit » (Proust, $1971: 381$ ) - le régime nocturne. Le mythe illustrant le thème de l'irrévocabilité de la mort le jour des noces, invite Moreau à utiliser le thanatisme sur le plan émotionnel pour illustrer à la fois son désespoir et la tristesse d'Orphée « en attendant l'aube » (Durand, 2016 [1960] : 197). À cet égard, il convient également de noter que le mot « cimetière », en français, doit son étymologie à koimêtêrion qui, en allusion à la tombe, comme l'indique Gilbert Durand (2016 [1960] : 248) dans le sillage de Jung (1932 [1912] : 28), « signifie chambre nuptiale ». Ce regard symbolique invoque

\footnotetext{
${ }^{9}$ La reprise de la mythologie biblique à travers la figure d'Orphée renvoie au poème de Victor Hugo dans La Légende des siècles où il prône une Eurydice « sacrée », adjectif qui attribue à la femme aimée un caractère divin et éternel (Hugo, 2002 [1859] : 342). Dans ce sens, la femme est renversée car celle-ci est considérée non comme la responsable du péché, mais de l'origine du monde et, à ce titre, sacrée.
} 
l'union sacrée et sentimentale entre ces personnages, en définissant le cimetière comme l'espace conciliateur de Thanatos et d'Éros. Le penchement sur la mort évoque chez Gustave Moreau l'idée mythique d'une réunion intime avec la bien aimée et, par conséquent, la promesse de survivre à Kronos. En outre, le XIX ${ }^{\mathrm{e}}$ siècle était encore fasciné par la mort, aussi, dans une perspective romantique, nous avons accès à la " mort de soi ", notion adoptée par Philippe Ariès (1977 [1974] : 46), selon laquelle la mort s'écarte du soi et se projette sur l'autre. Ainsi, étant la mort et l'amour deux entités isomorphiques très proches, Orphée et Eurydice - Moreau et Alexandrine, respectivement - restent unis dans une tombe. Une fois qu'Eurydice ou Alexandrine sont physiquement mortes, le poète et le peintre deviennent la projection de leur identité. Comme s'il était un effet illusoire, la mort « s'exprime mieux dans la double vue de l'anamorphose » (Hallyn, 1982 : 13), ce qui explique que chez Gustave Moreau, le paysage illustre la mort à partir d'un jeu d’images dédoublées et le syncrétisme iconographique du régime nocturne.

\section{Symboles et archétypes du paysage thanatique et féminin}

Comme expliqué précédemment, la mythocritique introduit une synthèse constructive entre les différentes critiques littéraires, artistiques et sociologiques, en focalisant le mythe comme une entité symbolique. Pour y accéder, la méthode consiste à rechercher des mythèmes redondants, à examiner les schémas combinatoires dans les contextes où ils se produisent, à traiter les différentes unités symboliques que le mythe exprime en les comparant avec des récits d'autres époques et civilisations spécifiques. En ce sens, la dimension psychique et féminine du paysage funéraire de Gustave Moreau est conforme aux valeurs d'une société occidentale, fortement déterminée par le christianisme (Ariès, 1977 [1974] : 23) et le regard romantique, dans une dimension esthétique, ontologique et spirituelle. Au-delà de ces facteurs, le paysage est défini sur la toile de ce peintre comme son memento mori, un lieu de transformation, "símbolo del inconsciente, símbolo maternal y femenino » (Cirlot, $1958: 455$ ), ainsi qu'en tant que paysage morphologique et symboliquement sexué. Contrairement au récit biblique, la mythologie occidentale est fortement marquée par un substrat matriarcal, perméable au discours littéraire et artistique, de sorte que Orphée sur la tombe d'Eurydice entraîne une approche herméneutique. Cette influence se dresse sous la figure d'Hermès-Mercure, celui qui connecte le masculin et le féminin, le rationnel et le tellurique, le monde souterrain nocturne des âmes et le monde diurne des mortels ; une dichotomie que nous allons creuser ciaprès.

\subsection{Du symbole maternel à l'archétype Hermès-Mercure}

Si Gustave Moreau a été souvent jugé comme « un homme à la mère » (Gauville 1998) ou un misogyne, il faut cependant noter qu'il accorde dans ses œuvres une place importante à la femme, aspect maintes fois remis en question dans un bassin sémantique décadent où l'androgyne se révèle 
l'un des symboles les plus présents dans l'esthétique de la fin de siècle. De même, Frédéric Monneyron (1993 : 76), dans son article « De l'androgyne au misogyne : l'infernale dialectique des décadents », souligne que la pensée androgyne du XIX ${ }^{\mathrm{e}}$ siècle se reflète « sous la forme de deux figures concurrentes : le jeune homme efféminé et la femme masculine, qui enveloppent tout le champ littéraire et artistique de la décadence ».

Premièrement, en ce qui concerne le symbole féminin, le paysage funéraire est dessiné comme une équation dans laquelle Orphée ressemble à une femme, ou plus précisément, à la mimésis de deux êtres si semblables l'un à l'autre qu'il est difficile de différencier le pôle masculin chez la femme (Animus) du pôle féminin chez l'homme (Anima). Cet Orphée féminisé renvoie à La mort d'Ophélie (1853) de Delacroix, ou à celle des naturalistes comme Ophélie (1881) de Jules-Bastien Lepage et Ophélie (1883) de Alexandre Cabanel, dans le sens que la figure humaine se fusionne avec l'humide et le végétal dans le milieu paysager. Or, cette double identité dans la toile de Moreau passe par une application de la symbolique alchimique à la mythologie à partir de deux archétypes : la lune - comme un élément féminin, en circonférence - et le tombeau - aspect masculin sous forme triangulaire.

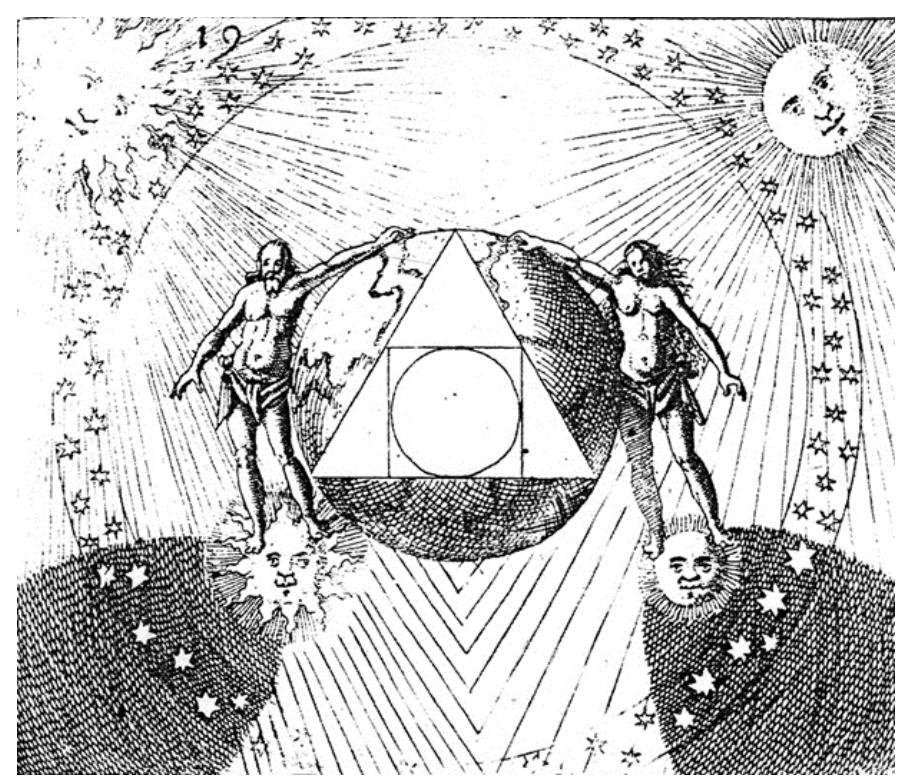

H. Jamsthaler. Viatorium spagyricum (quadrature du cercle), 1625. Gravure sur bois.

À partir de la théorie de Jung et de son disciple Pierre Solié, il est possible de prouver les deux oppositions symboliques proposées dans la toile et leur conversion en une seule image à travers la lune sphérique et le tombeau triangulaire. Dans l'imaginaire de Moreau, ces éléments sont destinés à refléter le désir d'Orphée de récupérer l'âme perdue d'Eurydice et d'obtenir son immortalité. Les deux symboles, insérés dans le contour carré de la toile, évoquent 
dans ce paysage le désir d'éternité si présent dans la pierre philosophale ${ }^{10}$. Ainsi, les trois figures mères - cercle, triangle et carré - ne font que se réduire à une seule, comme Jung soutien dans le Rosarium philosophorum : « Fais de l'homme et de la femme un cercle rond, et extrais-en un carré, et du carré un triangle. Fais un cercle rond et tu auras la pierre philosophale » (Jung, 2014 [1944] : 167). Cette communion introduit la possibilité d'équilibre des polarités dans le tableau. Les trois figures géométriques ainsi que les quatre personnages - Orphée et Eurydice, Gustave Moreau et Alexandrine Dureux - que nous allons évoquer dans une perspective à la fois mythologique et alchimique, au-delà d'être considérés des manifestations d'une structure ou d'une représentation symbolique, ils doivent être compris comme une totalité significative. En ce sens, les deux polarités deviennent des semblables.

En tenant compte de ces éléments, et en accordant une attention particulière au système des contraires qui constituent la scène dessinée par Gustave Moreau, l'architecture symbolique du tombeau propose diverses lectures : une possible porte aux Enfers et l'incarnation de l'Anima d'Orphée et de Christ, celui-ci « assimilé à Orphée porteur du bonnet thrace et de la lyre, héros fondamentalement initiatique dans sa démarche de transfiguration de l'homme pécheur, chargé de la faute d'Adam, en un homme nouveau » (Durand, $1997: 22$ ). En effet, le triangle sépulcral représente, comme le souligne Montoro Araque (2018: 241), « les trois divisions principales de l'univers - ciel, terre, enfers - ». Ces trois dimensions, parfaitement délimitées sur la toile, font allusion non seulement à l'endroit où repose le corps d'Eurydice, mais aussi à la figure d'Hermès Trismégiste, trois fois grand. Il ne faut pas oublier que le triangle reposant sur sa base est un symbole solaire, donc masculin :

On sait que, depuis toujours, les chiffres impairs sont masculins, et les chiffres pairs féminins, et ce non seulement ici, en Occident, mais aussi en Chine. De ce fait, la Trinité est une divinité expressément masculine, à laquelle l'androgynie du Christ, la position particulière et l'élévation de la mère de Dieu n'apportent pas de véritable contrepoids (Jung, 2014 [1944] : 50).

Cependant, l'élément funéraire qui prend forme dans cette œuvre est un écho à l'ambigüité de genre d'Orphée et du Christ, d'un point de vue mythologique et religieux, faisant appel à Daena et Shekhina, « l'apparence féminine de Dieu ", et en allusion à la Trinité ou à la Trimurti. La crypte triangulaire fait référence à la création, du point de vue masculin, car le numéro trois est le résultat de la conciliation entre deux opposés qui demeureraient

${ }^{10}$ Selon Louis Figuier (1860), parmi les propriétés fondamentales que les alchimistes attribuaient à la pierre philosophale, il se trouve la capacité de prolonger la vie ou d'atteindre l’immortalité. 
incompatibles s'ils ne rencontraient pas un terrain de conciliation. Celle-ci renvoie également à la création féminine, à une image de Dieu reflétant la méfiance envers les forces obscures d'une féminité tellurique et chtonienne. C'est probablement à l'origine d'un archétype thanatique qui conforme ces transmutations symboliques, que nous pouvons établir « la conjonction d'héros-gamos qui donnera lieu, en même temps, à l'unité androgyne du mythe hermétique » (Montoro, $2018: 241)$. En outre, si nous appliquons à Orphée sur la tombe d'Eurydice les principes de la pierre philosophale en alchimie, la figure androgyne d'Orphée se définit comme l'exemple maximal et antithétique des pôles opposés qui parviennent à se synthétiser à la perfection dans cette figure. Comme d'autres artistes et écrivains de son époque, chez Gustave Moreau l'androgyne peut rejoindre l'archétype Hermès-Mercure, " la dualité féminine que le héros cherche à atteindre " (Montoro, 2018 : 241). Gustave Moreau reprend ainsi deux pôles - en l'occurrence les deux archétypes de Jung, animus et anima - qui évoluent vers une convergence dans la tombe alchimique de cette toile, au moyen de la structure synthétique typique du régime nocturne de Durand. Cette union des contraires se traduit par « la vieille formule hermétique Un le Tout (En to Pan) » (Bonardel, 2011: 124). Moreau l'illustre ainsi, au sens où l'exprime Marcel Proust (1990 [1913] : 413), dont l'admiration pour le peintre le conduit à l'assimiler à Elstir, dans un portrait de Miss Sacripant, marqué par des traits d'ambigüité :

Le long des lignes du visage, le sexe avait l'air d'être sur le point d'avouer qu'il était celui d'une fille un peu garçonnière, s'évanouissait, et plus loin se retrouvait, suggérant plutôt l'idée d'un jeune efféminé vicieux et songeur, puis fuyait encore, restait insaisissable. Le caractère de tristesse rêveuse du regard, par son contraste même avec les accessoires appartenant au monde de la noce et du théâtre, n'était pas ce qui était le moins troublant.

La conciliation des contraires à partir des structures synthétiques des régimes de l'image nous permet d'appliquer à l'œuvre de Gustave Moreau « l'association de deux mots contraires » au sein d'une même unité, et c'est à travers "d'une même figure mythique, l'androgyne » que Gautier, l'un de ses contemporains, " se permet de rejoindre l'archétype Hermès-Mercure » (Montoro Araque, 2019 : 195). De même, à propos du caractère androgyne, Proust reprend les caractéristiques présentes dans la toile du peintre symboliste Poète indien (1898) et met en lumière la figure d'Orphée, la jeunesse, la poésie et le chant :

Il est plus beau peut-être encore que les autres, aux autres il est comme de belles paroles, le chant. Comme la poitrine entourée de roses du jeune chanteur, il est soulevé par l'enthousiasme. Mais il vient bien, comme les autres, du pays où les couleurs ont cette couleur, où 
les poètes ont un visage de femme et les insignes des rois, sont aimés des oiseaux, connus de leurs chevaux, couverts de pierres précieuses et de roses, c'est-à-dire où c'est l'allégorie qui est la loi des existences (Proust, 1971 [1954] : 674).

Nous pouvons ainsi affirmer que Gustave Moreau s'immerge dans un paysage thanatique féminisé pendant une période postromantique illustrant le thème de la mort ainsi que celui de l'hermaphroditisme. Parallèlement, quelques années plus tard, le peintre Louis Cattiaux dans Le Message Retrouvé ou l'Horloge de la Nuit et du Jour de Dieu (1956), évoque le Christ sous les qualités du dieu grec Apollon, représenté comme l'Orphée de Moreau, imberbe et avec des cheveux dorés en raison de son identification avec le soleil, métaphore de l'âge d'or. Son apparence renvoie également à la figure mythique de la sirène, personnage aquatique qui incarne la femme et l'esprit tellurien face à l'homme, fils du ciel (voir Wirth, 1961). L'alternance du masculin et du féminin, perméable à la poésie et au paysage, se définit à travers l'amour d'Orphée pour Eurydice, par « l'expression de l'élément masculin, le génie du poète, à la recherche de son principe féminin » (Kushner, $1960: 217)$.

À propos du rapport d'Hermès avec l'androgyne et la symbolique pierreuse du cimetière, selon la théorie de Franz-Karl Mayr, le dieu en tant que médiateur est très présent dans les cultures matriarcales méditerranéennes ainsi que dans la culture patriarcale hellénique. Tout en reprenant la thèse de Nilsson, le philosophe affirme que la plus ancienne forme de représentation d'Hermès est comme la pierre qui « se dresse sur les habituels tas de pierres qui, entassés par les passants, formaient des monuments funéraires » (Mayr, 1989 : 15). Pareillement, tout en s'appuyant sur l'onomastique et l'étymologie, Kárl Kerényi (1989 : 16) soutient que « Hermès est linguistiquement lié à "erma" (pierre). Tant son nom que son association à la pierre nous conduisent au culte ancestral de la Pierre Sainte, sous les deux aspects de "tombeau" et de "phallus" ". Cependant, de ce symbole thanatique - mais vaginal - de la Grande Déesse - à savoir la tombe d'Eurydice, symbole du ventre maternel qui suit les principes du régime nocturne -, Hermès émerge dans une dimension orphique et phallique. En ce sens, Mayr introduit la transition et l'osmose entre le premier Hermès chthonien, à notre avis, Orphée, serviteur de la Magna Mater - étant celle-ci incarnée par Eurydice - et celui qui sera le messager de Zeus, le grand père céleste. Ce passage qui marque la transition d'un Hermès primitif et matriarcal à un Hermès patriarcal porteur de la nouvelle culture d'Apollon et du progrès prométhéen tout au long de la période décadente, est également introduit par Gustave Moreau par le biais de l'archétype Hermès-Mercure, le symbole maternel et féminin et l'image dédoublée d’Orphée et Eurydice.

\section{2. Éléments végétaux et aquatiques dans la configuration my- thique du paysage}


Symbole de l'inconscient du peintre, Orphée s'agrippe à un arbre mort, élément antagoniste de l'arbre de vie ou de l'arbre mythique tenant à représenter un lien entre le monde visible et le monde intelligible. Ce symbole végétal - évoquant le cosmos vivant, ainsi que le principe d'ascension du monde profane et terrestre vers un autre monde céleste et supra-cosmique - fait référence au chagrin de l'artiste, ainsi qu'au corps d'Eurydice. À ce sujet, dans Les Métamorphoses d'Ovide, nous pouvons souligner une réminiscence aux "métamorphoses des femmes édoniennes" (Béague, 1998 : 57) qui deviennent des arbres : « Pourtant Lyaeus ne permet pas que ce crime reste impuni. Souffrant d'avoir perdu le chantre (uates) de son culte, il fixa aussitôt dans les forêts par une racine tortueuse toutes les femmes édoniennes qui avaient vu ce forfait sacrilège " (Mort d'Orphée. Châtiment des Ménades. 11, 67-70). Selon notre hypothèse, cet arbre représente Eurydice, car les dieux sont souvent assimilés à des fleurs, tandis que les nymphes, les dryades et les déesses comme Eurydice sont associées à des arbres. L'union d'Orphée et d'Eurydice - deux corps symboliques qui deviennent paysage dans l'huile de Gustave Moreau - embrasse la théorie de Françoise Frontisi-Ducroux (2017 : 13) autour des métamorphoses végétales dans les poèmes d'Ovide auxquelles " on pourrait ajouter le changement de sexe ». L'arbre, comme Hermès, est le conducteur de la terre et du cosmos, le point d'union où se rencontrent simultanément Moreau et Orphée, Alexandrine et Eurydice. Cet élément abrite donc les deux pôles, le ciel et la terre, le masculin et le féminin.

Dans ce contexte, il convient de remarquer que l'arbre mythique se rattache au principe féminin et masculin, lunaire et solaire. Symbole omniprésent dans cette œuvre, l'arbre meurt et renaît sans cesse en lien avec l'union amoureuse, voire dans les représentations chrétiennes du mariage mystique, tel que Victor Hugo (1837 [1831] : 38) l'exprime, «l'amour c'est comme un arbre ». Dans l'iconographie médiévale, des images d'un Arbre de Vie mâle et femelle sont récurrentes en Occident comme en Orient. Cependant, la symbolique de l'arbre possède une ambivalence importante car il introduit une image androgyne ${ }^{11}$. Du point de vue masculin, le tronc de l'arbre qui s'élève vers le ciel renvoie à une force et à une puissance phalliques et solaires qui fécondent le monde. En revanche, sous une symbolique féminine, l'arbre porteur d'une frondaison épanouie, l'arbre fruitier ou l'arbre creux s'identifie à une matrice par leur identification à la condition humaine, à la naissance et à la mort. Il s'agit du principe féminin de gestation lunaire et fertile. Très probablement inspiré de Hugo, Gustave Moreau symbolise dans l'arbre sec un cœur en ruine : «C'est que l'amour est comme un arbre, il pousse de lui-même, jette profondément ses racines dans tout notre être, et continue souvent de verdoyer sur un cœur en ruine » (Cooke,

${ }^{11}$ Voir Les Arbres du Soleil et de la Lune de Jean Wauquelin, Chroniques d'Alexandre Bruges, XVe siècle, Marsailly/Blogostelle. 
2002 : 536). L'image de l'arbre exprime à la fois les principes cycliques d'ascension, de descente et de réciprocité, sur le plan universel comme sur le plan individuel. La disposition de ces symboles, très fréquents dans le paysage funéraire, nous situe devant une nouvelle métaphore : la tombe comme un arbre. Gustave Moreau nous livre un cimetière paysager, où l'occupation des plantes acquiert une plus grande importance que celle du minéral architectural sépulcral. Ainsi, dans cette peinture à l'huile, la nature non seulement comporte la vie mais aussi la mort, de sorte que le peintre symboliste décrit comment « le poète est tombé inanimé au pied de l'arbre sec, foudroyé par la foudre » et la façon dont « la lyre désespérée est suspendue aux branches gémissantes et douloureuses » (Cooke, 2002 : 134). Dans l'imaginaire de Gustave Moreau, le cimetière devient un lieu thanatique, où, accablé par le chagrin, il ressemble à un poète quiescent qui lutte contre Kronos et Thanatos, cherchant à retourner dans sa propre tombe, au ventre, au refuge souterrain et naturel qui est la caverne maternelle.

Enfin, en ce qui concerne le symbole maternel, il faut souligner la présence de l'eau dans le cimetière et la relation avec l'au-delà. Moreau écrit : « La lune apparaît au-dessus de l'édicule et de l'étang sacré » étant l'eau un «murmure de vie et d'espoir de la nature » (Cooke, 2002 : 134). L'univers aquatique présent dans ce paysage funéraire fait référence au retour aux eaux féminines, à l'eau amniotique. Aux dires de Durand (2016 [1960] : 109), " cet isomorphisme de la lune et des menstrues se manifeste dans de nombreuses légendes qui font de la lune ou d'un animal lunaire le premier mari de toutes les femmes ». En outre, l'étang, comme s'il s'agissait d'un sépulcre, repose dans la structure mystique du régime nocturne : d'un côté, la structure synthétique est régie par le temps psychique et, d'autre part, la structure mystique est caractérisée par l'euphémisation d'un temps destructeur qui s'annule au moyen d'images de protection et de repos, analogues au concept du retour au ventre maternel ; isotopies d'images contenantes et protectrices qui rassemblent « la dominante digestive » (Durand, 2012 : 208) et transformatrice. Contrairement au régime diurne, où la peur de la mort et de la descente est fatale, dans ce régime, cette action devient l'acte d'avaler et de renaître du ventre de la mère. En ce sens, la descente d'Orphée - qui n'est plus une chute morale - est liée à la structure mystique, à l'autonomisation, au développement et à la régénération. Cette descente est également liée à la régression dans la perspective de la psychanalyse jungienne. Parallèlement à l'imagination créatrice et libératrice de Moreau, dans Aurélia de Nerval, la descente aux enfers « représente une véritable purification », une plongée dans les profondeurs du psychisme pour se libérer « des images qui l'obsèdent en les exprimant » (Kushner, 1961: 77). Dans le même ordre d'idées, ayant cette huile une fonction canalisatrice, Renato Boccali fait référence au pouvoir libérateur d'Orphée - et dans ce cas-là, à Moreau - à travers le chant - et la peinture -, car il « veut voir une Eurydice nocturne mais, pour le faire, il a besoin de la ramener à la lumière et ainsi il 
la perd » (Boccali, 2016 [1960] : 61). La mort est ici euphémisée et comprise, comme le décrit Durand, en tant que « retour plus ou moins cénesthésique et viscéral » (2012: 208) teinté d'espaces de méditation, d'intimité et de transcendance. Comme Moreau le reflète à travers le cimetière et les images archétypales, le tombeau assimilé à Eurydice et à la porte des enfers ; au " monde intérieur avec sa pénombre utérine ", d'après la pensée de Peter Sloterdijk (2002 : 296). C'est la tombe en tant que « porte de la mère » qui, dans une dimension religieuse et mythique, témoigne ce système d'analogies : « La porte de la mère qui, en soi, sert de sortie, et uniquement de sortie, doit aussi être revendiquée comme une entrée - non point tant dans un acte sexuel et érotique [...] mais dans un sens religieux, engageant toute l'existence » (Sloterdijk, 2002 : 296).

D'un côté, la présence de l'eau dans le cimetière répond à une autre dualité : la vie et la mort. Selon Jung et les alchimistes, aqua est spiritus, l'eau étant donc conçu comme l'esprit d'Eurydice et d'Alexandrine Dureux dans ce paysage funéraire. Ces valeurs symboliques et archétypales opposées sont introduites par le psychanalyste qui affirme qu'il faut « suivre le chemin de l'eau qui descend toujours » (Jung, 1995 [1954] : 30), chemin qu'Orphée a suivi lorsqu'il a sauvé Eurydice des eaux du monde souterrain, dans le lac Styx, puisque, comme le soutien Paul Claudel (1934 [1928] : 235), « tout ce que le cœur désire peut toujours se réduire à la figure de l'eau ». Ainsi, l'eau en tant que « matière du désespoir », aux dires de Bachelard, est similaire à la conception de Durand des eaux de l'Achéron comme des « séjours de tristesse » (Durand, 2016 [1960] : 84).

D’un autre côté, nous pouvons souligner une relation symbolique entre l'eau et la lune, déterminée par le symbole féminin de la menstruation, et que Mircea Eliade (1952 : 64) met en exergue à travers « les puissances sacrées concentrées dans les Eaux, dans la Lune, dans la Femme ». Toutes les trois sont régies par la même divinité, Vénus ou Aphrodite, et elles sont liées par la fertilité, toujours aqueuse sous l'influence des cycles lunaires, car cet astre est une épiphanie dramatique du temps suspendu lié à la mort. L'idée de fécondité se rapporte aussi à l'arbre mythique - ou arbre de vie cité précédemment ; un arbre cosmique qui divise le ciel et la terre, le monde sacré, divin ou métaphysique et le monde réel. La fertilité symbolique de l'arbre - naturelle ou spirituelle - se marie souvent à celle de l'eau. Dans l'eau épaisse de l'étang se trouve l'expression physiologique de la féminité, et donc d'Eurydice. De plus, tant dans la descente aux enfers d'Inanna que dans celle d'Orphée, apparaissent des figures féminines et la quête incessante de l'amour, thèmes du romantisme qui se reflètent constamment dans le cimetière de Moreau.

Or, pour Virgile, le héros « se retourna pour voir, alors qu'elle atteignait presque la lumière, sa chère Eurydice » (Béague, 1998 : 46). En ce sens, la combinaison de la katábasis - la descente aux enfers - avec la 
nékya - l'évocation des morts - est reprise dans Orphée sur la tombée d'Eurydice ainsi que dans les structures diurnes de l'imaginaire. Celles-ci regroupent des symboles nyctomorphes - dimension temporelle des ténèbres liée au paysage crépusculaire - et catamorphes - des symboles de la chute qui représentent la descente aux Enfers du héros nocturne. En revanche, le régime nocturne comporte dans cette œuvre le symbole d'inversion qui entrainent des images redoublées, comme celle d'Orphée et celle d'Eurydice, ou de Moreau et d'Alexandrine, respectivement. Du point de vue de l'imagination symbolique, la transformation d'Orphée et d'Eurydice se traduit par l'évolution du régime diurne au régime nocturne, par le passage de la libido au symbole maternel, par le dépassement du temps mortel, de la descente à l'ascension. En d'autre termes, par « un voyage sans retour », car " jamais deux fois l'on ne se baigne dans le même fleuve et les rivières ne remontent point à leur source. L'eau qui coule est la figure de l'irrévocable » (Durand, 2016 [1960] : 98). Le mythe d'Orphée peut être lu à partir de cette transition, puisque en regardant Eurydice comme « Éros-Cupidon aux yeux bandés » (Durand, 2016 [1960] : 79) - et assimilée à la libido - Orphée la perd irrémédiablement :

Tantôt au contraire la libido composera avec les douceurs du temps, renversant comme de l'intérieur le régime affectif des images de la mort, de la chair et de la nuit, c'est alors que l'aspect féminin et maternel de la libido sera valorisé, que les schèmes imaginaires vont s'incurver vers la régression et la libido sous ce régime se transfigurera en un symbole maternel (Durand, 2016 [1960] : 201).

Ainsi, les eaux renvoient souvent aux profondeurs de l'inconscient et représentent le flux du temps et la féminité. L'eau en tant que symbole du devenir temporel est primordial dans Orphée sur la tombe d'Eurydice à partir de l'étang. L'inclusion de cet élément aquatique reflète le caractère hermésien et adjuvant de l'eau, puisque c'est dans le lac Styx qu'Hermès apparaît, de même qu'Orphée, en tant que psychopompe, " celui qui conduit les âmes des morts » (Brunel, 2000 : 13). L'image de l'étang dans cette œuvre représente aussi un symbole de la mort et de la féminité, car comme Jung (1995 [1954] : 148) l'affirme, "l'eau est ce qui tue et ce qui donne la vie ». De même, les eaux maternelles ou matritamah, ainsi que les eaux femelles dans les Enfers, sont réhabilitées dans ce jeu de transformations et d'oppositions propres de la configuration mythique et archétypale du paysage féminin chez Gustave Moreau.

\section{Conclusions}

À l'unisson du deuil, Gustave Moreau peint et écrit des paysages qui abritent des émotions où le sacré et le profane dessinent l'espace incarné pour « se livrer », cependant, « tout entier à la manifestation du rêve et de 
l'immatériel » (Cooke 2002 : 131). Le peintre postromantique et pré-symboliste codifie le mythe dans une œuvre non seulement artistique, mais aussi littéraire. Il personnifie la figure du poète qui pleure la mort de la nymphe Eurydice et d'Alexandrine Dureux, celles-ci symbolisées dans le paysage à partir des éléments aquatiques, sépulcraux, cosmologiques et végétaux. Ainsi, l'œuvre Orphée sur la tombe d'Eurydice répond à une double identité dans la mesure où elle est intégrée dans l'ensemble des représentations multiples inhérentes aux mythes. En effet, les deux figures mythologiques présentes dans ce récit sont unifiées dans une totalité. Ces éléments nous rapprochent également d'un mythe qui devient symbole chez Moreau. La relation de ces deux personnages matérialise un processus d'intégration de leurs identités et, surtout, donne un sens à ces relations fusionnelles. De plus, à côté de la dimension féminine et maternelle du paysage thanatique, nous avons constaté la ressemblance entre Orphée et Hermès, deux personnages mythologiques permettant d'interpréter le caractère redondant des symboles et des archétypes.

Enfin, le paysage thanatique et féminin sous l'une des pôles et des variantes analysées ci-dessus tend dans l'œuvre de Gustave Moreau à la postulation d'une image du paysage qui se fait corps, encore plus large et plus universelle qui, par conséquent, correspond à celle de l'image archétypale. En d'autres termes, il est possible de se rapprocher d'une figuration idéale de la féminisation du paysage en lien avec Thanatos. Tous les éléments qui constituent cet archétype dans les compositions artistiques et les écrits de Gustave Moreau, issus de la génération romantique, sont intégrés dans le régime nocturne durandien - un schéma d'images opposées -, et introduisent une nouvelle perspective dans l'étude du paysage des mythes grecs. Parallèlement, les mythes personnels repris par cet artiste révèlent le caractère dynamique d'un archétype que Moreau a fait sien et qui a consolidé le paysage le plus singulier dans le champ pictural de la fin du XIX ${ }^{\mathrm{e}}$ siècle. Outre son halo romantique, le lien entre symbolisme et onirisme témoigne l'hommage que l'auteur rend à la nature, à la mort et à l'amour, ainsi qu'aux êtres qui se fondent dans des architectures fantastiques et paysagères, aux analogies alchimiques et aux éléments végétaux sexués. Ce monde peuplé de symboles introduit des tendances allégoriques dans l'expression de formes artistiques et symboliques inhérentes à ce qui est féminin.

Orphée sur la tombe d'Eurydice est une composition dotée d'une pensée mythique où se recréent l'imagination de l'artiste et son regard le plus intime, tout en reflétant dans le paysage les grands thèmes d'un romantisme tardif qui anticipe les nouvelles pratiques et les attentes du monde contemporain. À travers la peinture, Moreau permet au mythe de perdurer, au sens où l'exprime Gilbert Durand. Il démontre, ainsi, la nécessité de prendre en compte l'évolution du paysage dans l'histoire des représentations religieuses et mythologiques du dix-neuvième siècle à nos jours. 


\section{RÉFÉRENCES BIBLIOGRAPHIQUES}

ARIÈS, Philippe (1977 [1974]) : Essais sur l'histoire de la mort en Occident du Moyen Âge à nos jours. Paris, Seuil.

BACHELARD, Gaston (1948) : La Terre et les rêveries de la volonté. Essai sur l'imagination de la matière. Paris, Éditions José Corti.

BACHELARD, Gaston (1984) : La poétique de la rêverie. Paris, Presses Universitaires de France.

BAUDRILLARD, Jean (1987) : "Au-delà du vrai et du faux ou le malin génie de l'image ». Cahiers internationaux de Sociologie, 82, 139-145.

BÉAgue, Annick; Jacques Boulogne ; Alain DeREMETZ \& Françoise Toulze (1998) : Les Visages d'Orphée. Villeneuve d'Ascq, Presses universitaires du Septentrion.

BOCCALI, Renato (2016 [1960]) : "Le regard d'Orphée ou la vision iconoclaste ». Poli-femo. Nuova serie di Lingua e Letteratura, 11-12, 53-68.

BONARDEL, Françoise (2011) : La voie hermétique. Paris, Dervy.

BRAGA, Corin (2019) : Archétypologie postmoderne. D'Edipe à Umberto Eco. Paris, Honoré Champion.

BRISSON, Adolphe (1899) : "Promenades et visites. L'ami du peintre ", in Le Temps. Portraits intimes. Paris, Armand Colin, vol. III, 89-100.

BRUNEL, Pierre (1992) : Mythocritique : Théorie et parcours. Paris, Presses Universitaires de France.

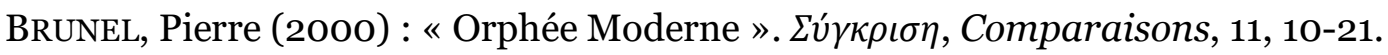

CharbonneaU-LaSSAY, Louis (1925) : " Le Christ-Orphée de Loudun ». Bulletin de la Société des Antiquaires de l'Ouest et des musées de Poitiers, 7 : 26, 125129

CIRLOT, Juan Eduardo (1958) : Diccionario de símbolos tradicionales. Barcelone, Luis Miracle.

Claudel, Paul (1934 [1928]) : Positions et Propositions. Paris, Gallimard.

COOKE, Peter (2002) : Écrits sur l'art par Gustave Moreau, sur ses œuvres et sur lui-même. Théorie et critique d'art. Fontfroide le haut, Éditions Fata Morgana.

CORBIN, Henri (1983 [1964]) : « Mundus imaginalis ou l'imaginaire et l'imaginal ». Cahiers internationaux du Symbolisme, 6, 3-26. Repris dans Face de Dieu, face de l'homme. Herméneutique et soufisme. Paris, Flammarion.

DELACROIX, Eugène (1986 [1829]) : Des critiques en matière d'art. Paris, L'Échoppe.

DELCROIX, Maurice \& Fernand HALLYN (1982) : Thanatos classique : cinq études sur la mort écrite. Paris, Éditions Jean-Michel Place. 
DURAND, Gilbert (2016 [1960]) : Les structures anthropologiques de l'imaginaire. Paris, Dunod.

DURAND, Gilbert (1979) : Figures mythiques et visages de l'œuvre. Paris, Berg international.

DURAND, Gilbert (1996a) : Champs de l'imaginaire. Grenoble, Ellug.

DURAND, Gilbert (1996b) : Science de l'homme et tradition. Le nouvel esprit anthropologique. Paris, Albin Michel.

DURAND, Gilbert (1997) : « Les Nostalgies d'Orphée, petite leçon de mythanalyse », Religiologiques, 15 [Metka Zupancic, éd., Orphée et Eurydice : mythes en mutation], 21-41.

DURAND, Gilbert (2003) : Mitos y sociedades: introducción a la mitodología. Buenos Aires, Biblos.

ELIADE, Mircea (1952) : Images et symboles. Essais sur le symbolisme magicoreligieux. Paris, Gallimard.

FAIVRE, Antoine (1994) : « Perspectives hermésiennes », in Pierre Brunel, Dictionnaire des mythes littéraires. Monaco, Rocher, 757-759.

FIGUIER, Louis (1860) : «Principes fondamentaux de l'alchimie. Propriétés attribuées à la pierre philosophale », in Louis Figuier, L'alchimie et les alchimistes : Essai historique et critique sur la philosophie hermétique. Paris, L. Hachette \& Cie.

FRAISSE, Luc (2002) : « Les Lectures inspiratrices de Proust sur Gustave Moreau », in Pascal Dethurens (coord.), Une amitié européenne : nouveaux horizons de la littérature comparée. Paris, Champion, 82-132.

FroNTISI-DUCROUX, François (2017) : Arbres filles et garçons fleurs. Métamorphoses érotiques dans les mythes grecs. Paris, Le Seuil.

GAUTHIER, Patrick (1970) : " Proust et Gustave Moreau », in Europe, 19, 237-241.

GAUVILLE, Hervé (1998) : « Moreau, un homme à la mère. Immaturité et misogynie à Paris, une rétrospective recadre le peintre symboliste ». Libération, 2 octobre. URL : https://www.liberation.fr/culture/1998/10/o2/moreau-unhomme-a-la-mereimmaturite-et-misogynie-a-paris-une-retrospective-recadre-le-peintre-symbol_249757

GIRAUD, Jean-Pierre (2005) : « Typologie des mythes », in Danièle Chauvin, André Siganos \& Philippe Walter (dirs.), Questions de mythocritique. Dictionnaire. Paris, Imago, 359-370.

GODEAU, Florence (2008): "Mémoire littéraire, imaginaire paysageant : l'exemple des lectures "proustiennes" du paysage ». Les Cahiers de la Compagnie du Paysage, 5 [Odile Marcel, dir., Paysage visible, paysage invisible : la construction poétique du lieu], 113-123.

GuHL, Marie-Cécile (1972) : "Les Paradis ou la configuration mythique et archétypale du refuge ». Circé 3, Cahiers du Centre de Recherche sur l'Imaginaire, 11-104.

Hugo, Victor (1837 [1831]) : Notre-Dame de Paris. Bruxelles, Société Belge de Librairie.

HuGO, Victor (2002 [1859]) : La Légende des siècles. Paris, Gallimard. 
Jung, Carl Gustav (1932 [1912]) : Métamorphoses et symboles de la libido. Paris, Montaigne.

JUNG, Carl Gustav (2014 [1944]) : Psychologie et alchimie. Traduit de l'allemand et annoté par Henry Pernet et le docteur Roland Cahen. Paris, Buchet/Chastel.

JUnG, Carl Gustav (1995 [1954]) : Les Racines de la conscience. Études sur l'archétype. Paris, LGF Le Livre de Poche.

KUSCHNER, Eva (1961) : Le mythe d'Orphée dans la littérature française contemporaine. Paris, Nizet.

LITTELL, Jonathan (2006) : Les Bienveillantes. Paris, Gallimard.

LOSADA GoYA, José Manuel \& Antonella LiPSCOMB (2013) [coords] : Mito e interdisciplinariedad. Los mitos antiguos, medievales y modernos en la literatura y las artes contemporáneas. Bari, Levante Editori.

MAINGON, Claire (2015) : «Magie du funéraire : regard sur l'historiographie de la sculpture funéraire et l'image de la mort (XVII $-\mathrm{XX}^{\mathrm{e}}$ siècle) ». Perspective, $1,177-183$.

MARTIN, Jean (2002) : «Sur le sens réel des mots catastérisme et catastériser

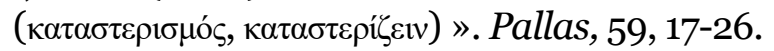

MAYR, Franz-Karl (1989) : La mitología occidental. Barcelona, Anthropos.

MONNEYRON, Frédéric (1993) : « De l'androgyne au misogyne : l'infernale dialectique des décadents ». Les Cahiers du GRIF, 47 [Misogynies], 75-86.

MONTORO ARAQUE, Mercedes (2018) : Gautier, au carrefour de l'âme romantique et décadente. New York, Peter Lang.

Murray SCHAEFER, Raymond (1979) : Le paysage sonore. Traduit par Sylvette Gleize. Paris, J-C. Lattès.

OvidE (2008): Métamorphoses, Livre X. Traduction et notes de Anne-Marie Boxus et Jacques Poucet. Bruxelles, Bibliotheca Classica Selecta - UCL (FIAL).

PRAZ, Mario (1977) : La chair, la mort, le diable. Le romantisme noir. Traduit par C.T. Pasquali. Paris, Denoël.

PROUST, Marcel (1971 [1954]) : " Notes sur le monde mystérieux de Gustave Moreau », in Contre Sainte-Beuve, précédé de Pastiches et mélanges et suivi de Essais et articles. Paris, Gallimard, 667-674.

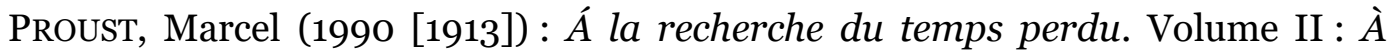
l'ombre des jeunes filles en fleurs. Paris, Gallimard.

SARDA, Marie-Anne (2004) : Gustave Moreau, paysages de rêve. Reims et Versailles, Musée des Beaux-Arts de Reims et Éditions Artlys.

SLOTERDJIK, Peter (2002) : Bulles. Sphères I. traduit par Olivier Mannoni. Paris, Pauvert.

STEIN, Leopold (1967) : «Introducing not-self ». Journal of Analytical Psychology, 12, 97-114.

Von Holten, Ragnar (1960) : L'art fantastique de Gustave Moreau. Paris, JeanJacques Pauvert. 
WIRTH, Oswald (1961) : Le symbolisme hermétique dans ses rapports avec l'alchimie et la franc-maçonnerie. Paris, Dervy.

WUNENBURGER, Jean-Jacques (2005) : «Création artistique et mythique » in Danièle Chauvin, André Siganos \& Philippe Walter (dirs.), Questions de mythocritique. Dictionnaire. Paris, Imago, 69-100. 